\title{
MicroRNA-375 targets the 3-phosphoinositide-dependent protein kinase-1 gene in pancreatic carcinoma
}

\author{
SHI-DUO SONG ${ }^{1 *}$, JIAN ZHOU ${ }^{1 *}$, JIN ZHOU ${ }^{1}$, HUA ZHAO $^{1}$, JIAN-NONG CEN $^{2}$ and DE-CHUN LI ${ }^{1}$ \\ ${ }^{1}$ Department of General Surgery, The First Affiliated Hospital of Soochow University; \\ ${ }^{2}$ Jiangsu Institute of Hematology, Soochow University, Suzhou, Jiangsu 215006, P.R. China
}

Received January 24, 2013; Accepted June 27, 2013

DOI: $10.3892 / \mathrm{ol} .2013 .1510$

\begin{abstract}
Pancreatic carcinoma (PC) is an aggressive malignancy with one of the poorest mortality rates. It is the sixth leading cause of mortality from malignant disease in China and the fourth leading cause of cancer-related mortality in the United States. The poor outcome reflects the requirement for an improved understanding of the transcriptional control of oncogenic signaling pathways. 3-phosphoinositide-dependent protein kinase-1 (PDK1) is a potent oncogenic driver of PC. The present study aimed to elucidate the transcriptional regulation of microRNA (miR)-375-targeted PDK1. miR-375 is a putative target and, in the present study, was observed to be significantly downregulated in the tumor compared with non-tumor tissues from patients with PC $(n=44)$. As determined by a luciferase reporter assay, the ectopic expression of miR-375 was identified to diminish the transcriptional activity of PDK1. Furthermore, immunoblotting revealed that miR-375 suppressed endogenous PDK1 protein levels. Functional assays showed that miR-375 was able to inhibit proliferation and promote apoptosis of the PC cells. miR-375 is a significant regulator of the $\mathrm{PDK} 1$ oncogene, suggesting that it may have a potential therapeutic role in the treatment of PC.
\end{abstract}

\section{Introduction}

Pancreatic carcinoma (PC) is an aggressive malignancy with one of the poorest mortality rates. It is the sixth leading cause of mortality from malignant disease in China and the fourth leading cause of cancer-related mortality in the United States (1-3). The estimated mortality is almost equal to the estimated incidence, with an overall 5-year survival

Correspondence to: Dr De-Chun Li, Department of General Surgery, The First Affiliated Hospital of Soochow University, 188 Shi Zi Avenue, Suzhou, Jiangsu 215006, P.R. China.

E-mail:dechunli@126.com

*Contributed equally

Key words: microRNA, microRNA-375, pancreatic carcinoma, 3-phosphoinositide-dependent protein kinase-1 rate of $<5 \%$ (3). Therefore, the identification of new associated factors and novel therapeutic targets for PC remains an imperative clinical issue. K-ras, CDKN2A, p53, DPC4 and 3-phosphoinositide-dependent protein kinase-1 (PDK1) are known to be mutated or inactivated during PC tumorigenesis, and mediate other functional and relevant cancer pathways (4-7). PDK1 is a key component in phosphatidylinositol 3-kinase-Akt-mammalian target of rapamycin (PI3K-Akt-mTOR) signaling, a well-documented pathway that regulates cancer cell survival and proliferation (7). PDK1 is a pleckstrin homology $(\mathrm{PH})$ domain-containing protein that is activated following PI3K activity, which in turn phosphorylates Akt1 at threonine 308 (or cognate locations on other isoforms) along with a large variety of other AGC kinase substrates. Although this kinase has a significant role in PI3K-Akt-mTOR signaling, the activating mutations of the gene encoding PDK1 have not been described. The deregulation of PDK1 is critical to the progression of pancreatic tumors.

MicroRNAs (miRNAs/miRs) are non-coding RNAs that consist of 21-23 nucleotides and are a recently emerging class of endogenous negative regulators of gene expression that possess a remarkable evolutionary conservation $(8,9)$. miRNAs are believed to modulate gene expression at the post-transcriptional level $(10,11)$. These small molecules exert their regulatory effects by base-pairing to partially complementary mRNA and functioning by two mechanisms, the degradation of target mRNA transcripts or the inhibition of mRNA translation $(11,12)$. miRNAs are also associated with the main phenotypes of numerous cancer cells (including PC), such as proliferation, invasion and apoptosis (13-15). Therefore, studying the functions and mechanisms of miRNAs may lead to new approaches for the categorization, diagnosis and treatment of human cancers. miR-375 is differentially expressed in various neoplasms (16-18). The effects of miR-375 may be cell-type specific (16). Few studies of PC have focused on targeting the clinical and prognostic significance of miR-375.

Our previous study identified that miR-375 is involved in PC through the suppression of PDK1 (19). However, the detail of how miR-375 regulates PDK1 expression in $\mathrm{PC}$ remains unknown.

The present study suggests that miRNAs may play key roles in tumorigenesis by regulating the expression of genes that are associated with oncogenic signaling pathways. 
However, further studies are required in order to explore and validate whether the putative target, miR-375, is involved in the regulation of the PDK1 oncogene.

\section{Materials and methods}

Tissue samples. PC tissues and their respective adjacent normal tissues were obtained post-operatively from 44 patients in 2009 from the Department of General Surgery, The First Affiliated Hospital of Soochow University (Suzhou, Jiangsu, China). Informed consent was obtained from all patients for their tissues to be used for scientific research. Approval for the study was obtained from the Department of General Surgery, The First Affiliated Hospital of Soochow University. All diagnoses were based on pathological and/or cytological evidence. The histological features of the specimens were evaluated by two senior pathologists according to the classification criteria from the World Health Organization (20). The tissues were obtained from the patients prior to chemotherapy or radiation therapy. The specimens were immediately frozen and stored at $-80^{\circ} \mathrm{C}$ prior to the microarray and quantitative (q)PCR analyses.

Cell culture. The human PC cell line, Panc-1, was maintained in DMEM supplemented with $10 \%$ FBS. The cells were cultured in an incubator at $37^{\circ} \mathrm{C}$ with $5 \% \mathrm{CO}_{2}$.

$q P C R$. Total RNA was extracted from the patients or the cell line samples using TRIzol (Invitrogen, Carlsbad, CA, USA), according to the manufacturer's instructions. The miR-375 expression level was determined by qPCR using Taqman assay kits (Applied Biosystems, Foster City, CA, USA), with U6 small nuclear RNA as an internal normalized reference. For the quantification of PDK1 and $\beta$-actin, the extracted RNA was reverse transcribed to cDNA using an oligo(dT) 12 primer and Superscript II (Invitrogen). The primers for these genes are summarized in Table I. The relative expression levels of the primers were measured in triplicate on a Prism 7900 Real-Time PCR machine (Applied Biosystems), according to the manufacturer's instructions.

Vector construction. To generate a miR-375 expression vector (pcDNA 3.1-miR-375) containing a miR-375 precursor, a sequence was amplified using the following primers: Forward, 5'-CCGCTCGAGCAGATGCGTTCAGGTGAG-3' and reverse, 5'-CGGAATTCTGGCGGCGGCAGGTGCCTG-3'. The amplified region was cloned into the pcDNA $3.1^{+}$vector (Genechem, Shanghai, China) using XbaI and HindIII restriction sites and confirmed using DNA sequencing, as previously described (21).

The putative miR-375 binding site at the 3' UTR of PDK1 was cloned downstream of a CMV promoter-driven firefly luciferase cassette in a pcDNA 3.1 vector (Genechem). A mutant form of this luciferase construct was also generated using the PCR-based overlap-extension procedures, as reported previously (22).

Transfection. Chemically synthesized RNAs, including scramble, an miR-375 mimic and its inhibitor, were obtained from GenePharma (Shanghai,China). For transfection, the cells were grown on 24-well culture plates to $70-80 \%$ confluence. Following $24 \mathrm{~h}$, the cells were cotransfected with Renilla luciferase reporter (PRL; $0.1 \mu \mathrm{g}$ ), the previously constructed reporter plasmids $(0.5 \mu \mathrm{g})$ and chemically synthesized RNA $(0.5 \mu \mathrm{g})$ using Lipofectamine 2000 transfection reagent (Invitrogen). Subsequent to being incubated for $24 \mathrm{~h}$, the cells were harvested using lysis buffer for use in the luciferase assay. G418 was then used to screen the monoclonal cells.

Luciferase reporter assay. For the luciferase reporter assay, Panc-1 cells $\left(3 \times 10^{4}\right)$ were plated in a 24-well plate and then cotransfected with $400 \mathrm{ng}$ pcDNA $3.1-\mathrm{miR}-375$ or pcDNA 3.1 empty vector, $200 \mathrm{ng}$ wild-type or mutant luciferase construct and $40 \mathrm{ng}$ PRL-TK (Promega, Madison, WI, USA) using Lipofectamine 2000 (Invitrogen) according to manufacturer's instructions. The cells were collected at $48 \mathrm{~h}$ post-transfection and analyzed using the Dual-Luciferase Reporter Assay System (Promega, Fitchburg, WI, USA). The pRL-TK vector provided the constitutive expression of Renilla luciferase and was used as an internal control to correct the differences in the transfection and harvest efficiencies (23). Each treatment was performed in triplicate and repeated in three independent experiments.

Western blotting. The treated cells were washed twice with ice-cold PBS, then directly lysed with $200 \mu 12$ X SDS cell lysis buffer in each well of a 6 -well plate cluster. The lysates were boiled, centrifuged at $15,680 \mathrm{xg}$ and loaded onto a $12 \%$ SDS-PAGE gel. The samples were electrophoresed for $4 \mathrm{~h}$ and transferred to a Millipore Immobilon transfer membrane (Millipore, Billerica, MA, USA) in Bio-Rad blot apparatus (Bio-Rad, Hercules, CA, USA). Subsequent to being blocked with 5\% skimmed milk in PBS-Tween-20 for $1 \mathrm{~h}$ at room temperature, the membranes were blotted with a goat polyclonal anti-hPDK 1 primary antibody overnight at $4^{\circ} \mathrm{C}$. The membranes were washed with TBST prior to being incubated with mouse polyclonal anti-goat secondary antibody linked to horseradish peroxidase (dilution, 1:2000) for $1 \mathrm{~h}$ at room temperature. The membranes were washed again with TBST and the blot was incubated in a detection reagent (ECL Advance Western blotting detection kit; Amersham Bio-science, Freiburg, Germany) and exposed to a Hyperfilm ECL film (Pierce, Rockford, IL, USA). Anti-PDK1 (Santa Cruz Biotechnology Inc., Santa Cruz, CA, USA) and GAPDH (KangChen Bio-Tech, Shanghai, China) antibodies were used in the western blot analyses.

Cell proliferation. The cells were seeded in a 96-well plate at a density of 10,000 cells per well and incubated for $96 \mathrm{~h}$. The in vitro growth was measured using Cell Counting kit-8 (CCK-8; Dojindo Laboratories, Kumamoto, Japan). The optical density (OD) at $450 \mathrm{~nm}$ was measured using a Microplate Reader (Bio-Rad) and the proliferation index was calculated as the experimental OD value/control OD value. A total of three independent experiments were performed in quadruplicate.

Cell cycle analysis. The cells were washed three times using cold PBS and then fixed in $70 \%$ ethanol in PBS at $-20^{\circ} \mathrm{C}$ for $2 \mathrm{~h}$. Following fixation, the cells were washed with cold PBS 
Table I. Primer sequences for qPCR analysis.

\begin{tabular}{lll}
\hline Gene & \multicolumn{1}{c}{ Forward primer $\left(5^{\prime} \rightarrow 3^{\prime}\right)$} & Reverse primer $\left(5^{\prime} \rightarrow 3^{\prime}\right)$ \\
\hline PDK1 & GTGTAGATTAGAGGGATG & AAGGAATAGTGGGTTAGG \\
$\beta$-actin & CTCCATCCTGGTCTCGCTGT & GCTGTCACCTTCACCGTTCC
\end{tabular}

PDK1, 3-phosphoinositide-dependent protein kinase 1; qPCR, quantitative PCR.

and stained with $1 \mathrm{ml}$ propidium iodide (PI) staining buffer (MultiScience, Hangzhou, China), which contained $200 \mathrm{mg} / \mathrm{ml}$ RNase A and $50 \mathrm{mg} / \mathrm{ml} \mathrm{PI,} \mathrm{at} \mathrm{room} \mathrm{temperature} \mathrm{for} 30 \mathrm{~min}$ in the dark. The analyses were performed on a FACScan flow cytometer (Becton-Dickinson, Sunnyvale, CA, USA). The experiments were repeated three times.

Cell apoptosis. The quantification of the apoptotic cells was performed using the Annexin-V-FITC Apoptosis Detection kit (Invitrogen), according to the manufacturer's instructions. Early apoptotic cells were defined as Annexin-V-positive, PI-negative cells. The analyses were performed on a FACScan flow cytometer (Becton-Dickinson). The experiments were repeated three times.

Tumorigenicity assays in nude mice. Six-week-old female $\mathrm{BALB} / \mathrm{c}$ athymic nude mice were subcutaneously injected into the right armpit region using $1.5 \times 10^{6}$ cells in $0.15 \mathrm{ml}$ PBS. The following groups of mice ( $n=5$ per group) were tested: Group I (miR-375 mimics) was injected with Panc-1 cells transfected with miR-375 mimics; group II (Mock) was injected with Panc-1 cells transfected with negative control; and group III (Vec) was injected with Panc-1 cells alone. The tumor size was measured every 2 or 3 days using calipers. The tumor volume was calculated with the following formula: $\left(\mathrm{L} \mathrm{x} \mathrm{W} \mathrm{W}^{2}\right) / 2$, where $\mathrm{L}$ is the length and $\mathrm{W}$ is the width of the tumor. The mice were sacrificed at four weeks and the weights of the tumors were measured. All experimental procedures involving animals were in accordance with the Guide for the Care and Use of Laboratory Animals (NIH publication no. 80-23, revised 1996) and were performed according to the ethical guidelines for animal experiments at the Department of Clinical Immunology, Soochow University (Suzhou China).

Statistics. The differences between the groups were evaluated using the Student's t-test. $\mathrm{P}<0.05$ was considered to indicate a statistically significant difference.

\section{Results}

miR-375 is a candidate miRNA that regulates the expression of PDK1. Bioinformatic algorithms (TargetScan 4.2; Whitehead Institute for Biomedical Research, Cambridge, MA, USA) were used to identify the candidate miRNAs that targeted the 3' UTR region of the PDK1 gene, and to subsequently identify miR-375 for further studies (Fig. 1D).

A qPCR analysis of miR-375 and PDK1 was performed in 44 pairs of PC tumor and matched adjacent non-tumor tissues. The results show that miR-375 was significantly down-regulated in the PC tumor tissues (Fig. 1A), while PDK1 was upregulated in the PC tumor tissues (Fig. 1B). PDK1 was inversely correlated with miR-375 (Fig. 1C), suggesting that PDK1 is a target of miR-375.

PDK1 is a direct target of $m i R-375$. To confirm that PDK1 is a target of miR-375, a luciferase reporter assay was performed. The relative luciferase activity of the reporter that contained the wild-type 3' UTR was significantly suppressed when miR-375 was cotransfected (Fig. 2A). In contrast, the luciferase activity of the mutant reporter was unaffected by the cotransfection of miR-375 (Fig. 2A), indicating that miR-375 suppressed gene expression using the miR-375-binding sequence at the 3 ' UTR of the PDK1 gene.

The effect of miR-375 on the endogenous expression of PDK1 was further examined. The ectopic expression of miR-375 caused a decrease in PDK1 protein levels in the Panc-1 cells (Fig. 2B).

miR-375 suppresses PC cell proliferation. The significant reduction of miR-375 in the PC samples and its inhibitory effect on the PDK1 oncogene prompted an investigation into its possible biological role in cancer cells. To study the effect of miR-375 on cell proliferation, a CCK- 8 proliferation assay was performed.

The results showed that the proliferation of the pcDNA 3.1-miR-375-transfected cells was slower than the mock and empty vector-treated cells (Fig. 3).

miR-375 arrests the cell cycle at $G_{0} / G_{1}$ and induces $P C$ cell apoptosis. To determine the effect of miR-375 on apoptosis and the cell cycle of the PC cells, flow cytometry (FCM) was performed. The results reveal that the Panc-1 cells that were transfected with the miR-375 mimics had an evident cell cycle arrest at the $G_{0} / G_{1}$ phase in contrast with the mock and empty vector-treated cells (Fig. 4).

The results show that the apoptotic activities of the pcDNA 3.1-miR-375-transfected cells were higher than that of the mock and empty vector-treated cells (Fig. 5).

miR-375 suppresses tumorigenicity in vivo. To confirm the aforementioned findings, an in vivo tumor model was used. miR-375 mimic-transfected Panc-1 cells (miR-375 mimics), negative control-transfected Panc-1 cells (mock) and Panc-1 cells were injected separately into three groups of nude mice ( $n=5$ per group). At four weeks post-injection, the miR-375 mimics group had developed substantially smaller tumors than the other two groups (Fig. 6A). The tumor volume at the 
A

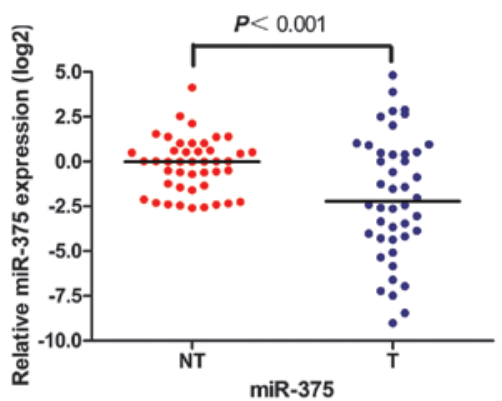

C

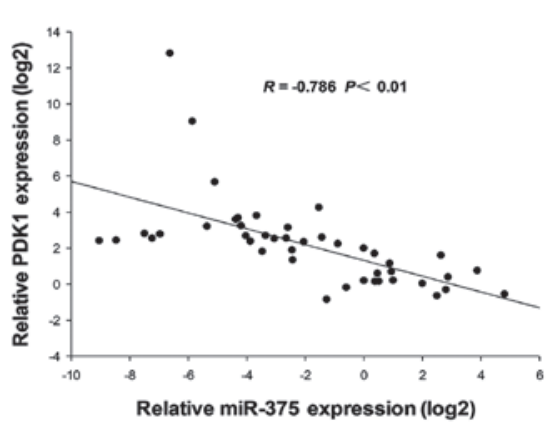

B

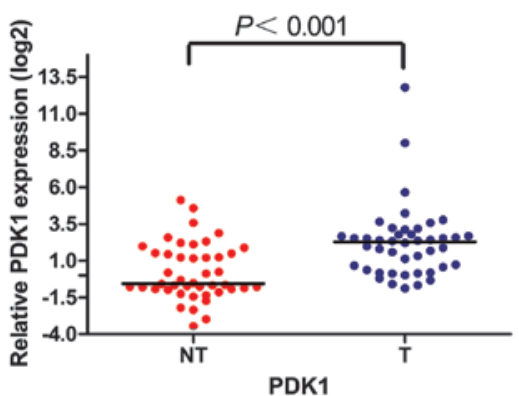

D

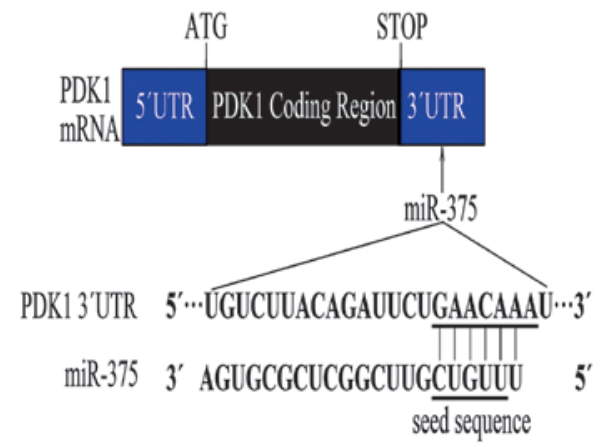

Figure 1. miR-375 is a candidate miRNA that regulates PDK1. (A) qPCR analysis showing the downregulation of miR-375 mRNA in the PC patients. (B) qPCR analysis showing the upregulation of PDK1 mRNA in the PC patients. (C) An inverse correlation of PDK1 and miR-375 expression levels was examined using a Spearman correlation analysis. (D) Schematic of the in silico analysis of the predicted binding sites showing that miR-375 binds to the 3' UTR region of PDK1 mRNA. The predicted consequential pairing between the target region (position 3490-3496 of PDK1 3' UTR) and the seed sequence of miR-375 is shown. PDK1, 3-phosphoinositide-dependent protein kinase-1; qPCR, quantitative PCR; miR-375, microRNA-375, PC, pancreatic carcinoma; NT, non-tumor; T, tumor.

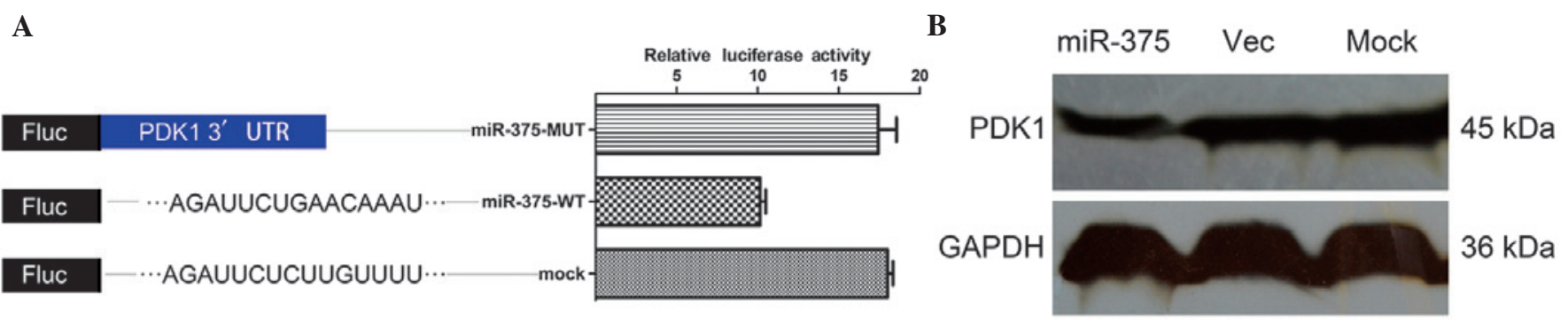

Figure 2. PDK1 is a direct target of miR-375. (A) PC cells were cotransfected with Renilla luciferase control and firefly luciferase reporter containing either wild-type (WT) or seed region mutated (MUT) PDK1 3' UTR and/or the pcDNA3.1-miR-375 (miR-375). (B) The cells were transfected with miR-375 or the pcDNA3.1 empty vector (Vec), and western blotting was used to show the suppressed expression of endogenous PDK1 in Panc-1 cells following miR-375 transfection $(\mathrm{P}<0.05)$. PDK1, 3-phosphoinositide-dependent protein kinase-1; miR-375, microRNA-375; PC, pancreatic carcinoma, Fluc, firefly luciferase.

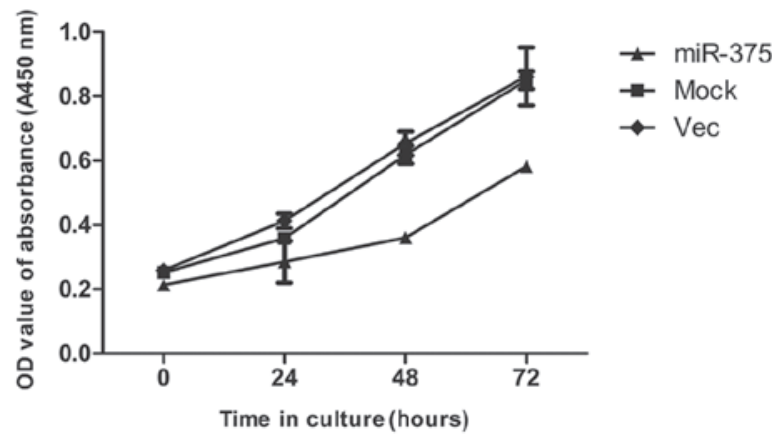

Figure 3. Association of miR-375 with the proliferation activities of PC cells. Ectopic expression of miR-375 was achieved by transfecting pcDNA3.1-miR-375. PC cells without any treatment (mock) and those that were transfected with pcDNA3.1 empty vector (Vec) were used as controls. A CCK-8 assay was performed to determine the cell proliferation activity. The data shown are the representative set of three independent experiments, and are expressed as mean \pm SEM. $\mathrm{P}<0.05$, relative to the mock control. miR-375, microRNA-375; PC, pancreatic carcinoma; OD, optical density; CCK-8, cell counting kit-8. 

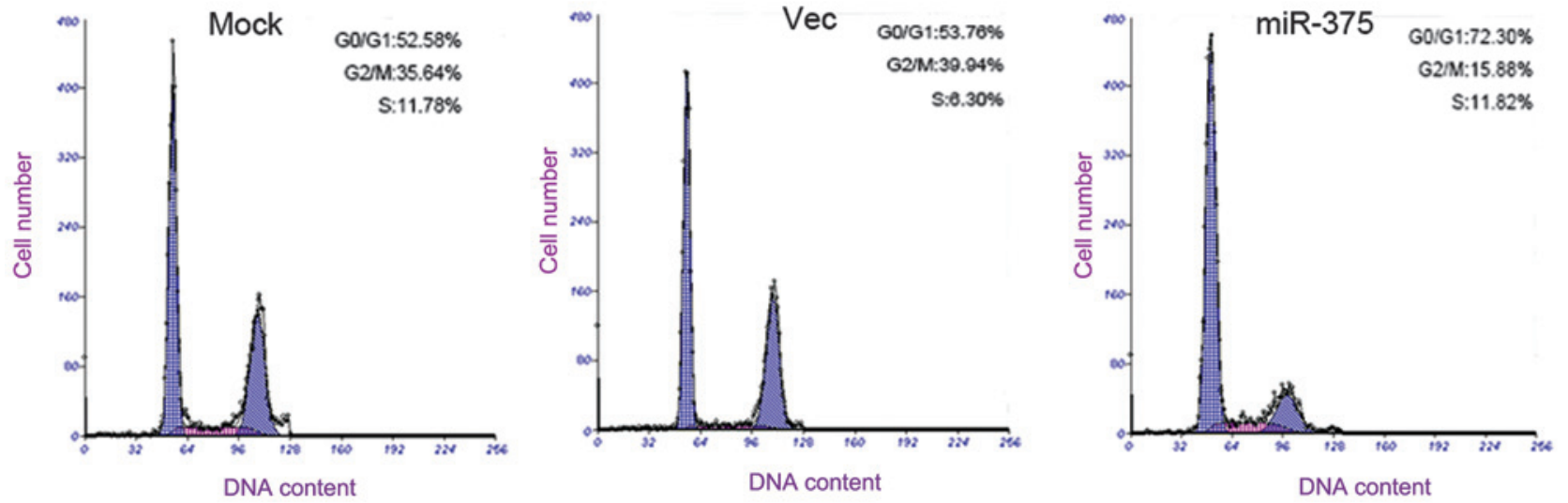

Figure 4. Association of miR-375 with the cell cycle of the PC cells. FCM was performed to determine the cell cycle. The data show that the PC cells that were transfected with miR-375 underwent a cell cycle arrest at the $\mathrm{G}_{0} / \mathrm{G}_{1}$ phase $(\mathrm{P}<0.05)$. PC, pancreatic carcinoma; FCM, flow cytometry; miR-375, microRNA-375.
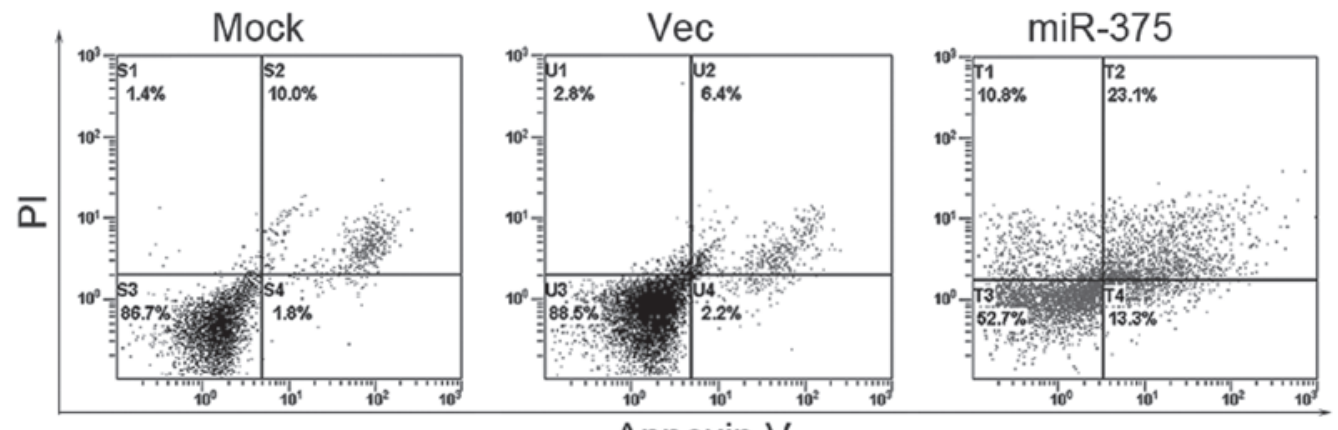

Annexin V

Figure 5. Association of miR-375 with the apoptosis of the PC cells. FCM was performed to determine cell apoptosis. The data show that the apoptotic activities of the pcDNA3.1-miR-375-transfected cells were higher than that of the mock and empty vector-treated cells $(\mathrm{P}<0.05)$. miR-375, microRNA-375; PC, pancreatic carcinoma; FCM, flow cytometry; PI, propidium iodide.
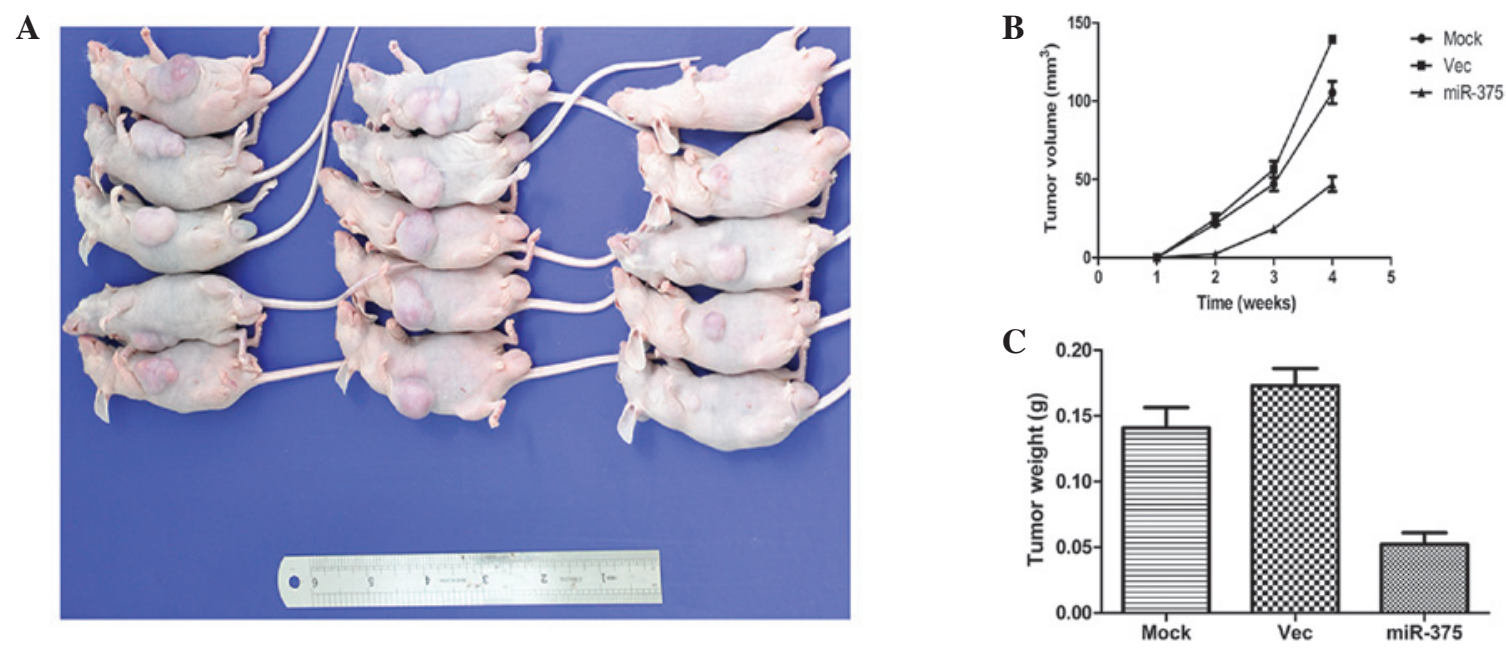

Figure 6. miR-375 suppresses tumorigenicity in vivo. Three groups of mice ( $\mathrm{n}=5$ per group) were tested. (A) The size of the tumors in three groups. (B) The tumor growth curves of the three groups for four weeks. (C) The mean tumor weight of the three groups at the end of the experiment. Data are presented as mean \pm standard deviation $(\mathrm{P}<0.05)$. miR-375, microRNA-375.

time of death of the mice that were injected with miR-375 mimic-transfected cells was $60.49 \pm 4.59 \mathrm{~mm}^{3}$, whereas the tumor volumes of the mice injected with mock or Panc-1 cells were $139.28 \pm 3.21 \mathrm{~mm}^{3}$ and $105.47 \pm 5.89 \mathrm{~mm}^{3}$, respectively
(Fig. 6B). Furthermore, the mean tumor weight at the end of the experiment was markedly lower in the miR-375 mimics group $(0.052 \pm 0.013 \mathrm{~g})$ compared with the mock and Panc-1 groups $(0.173 \pm 0.028 \mathrm{~g}$ and $0.141 \pm 0.031 \mathrm{~g}$, respectively; Fig. 6C). 


\section{Discussion}

The present study identified that miR-375 was expressed at a significantly lower level in PC tissues than in the respective adjacent normal tissues. In the human clinical specimens, the miR-375 mRNA levels were inversely correlated with those of PDK1. In addition, miR-375 directly regulated the expression of PDK1. The PDK1 oncogene was identified to be negatively regulated by miR-375 at the post-transcriptional level through a specific target site within the $3^{\prime}$ UTR of the gene. The overexpression of miR-375 may have resulted in the downregulation of PDK1. Genomic amplification of PDK1 and the loss of miR-375 may result in the enhanced expression of the PDK1 oncogene and, in turn, promote PC development, including the inhibition of cell proliferation, the induction of apoptosis and cell cycle arrest at $\mathrm{G}_{0} / \mathrm{G}_{1}$. PDK1 is a potent regulator in cell growth and a previous study supports its oncogenic role in mammalian cells (7).

Numerous growth factors activate the PI3K pathway, which in turn phosphorylates phosphatidylinositol-4,5-biphosphate (PIP2) to generate phosphatidylinositol-3,4,5-triphosphate (PIP3). An extensively studied signaling event controlled by PIP3 is the activation of a group of AGC family protein kinases, including isoforms of protein kinase $\mathrm{B}$ (Pkb/Akt) and the ribosomal S6 kinase (S6K), which play crucial roles in regulating the physiological processes that are relevant to metabolism, cellular growth, proliferation and survival $(24,25)$. PDK1 is a PH domain-containing protein that is activated following PI3K activity, which in turn phosphorylates Akt1 at threonine 308 (or cognate locations on other isoforms), along with a large variety of other AGC kinase substrates. Although this kinase has a significant role in PI3K-Akt-mTOR signaling, the presence of activating mutations of the gene encoding PDK-1 have not been described. A study by Westmoreland et al (7) suggested that PDK-1 expression levels may control the proliferation, survival and growth of developing pancreatic cells in PC, but this hypothesis has not been fully tested. PDK1 activation is primarily dependent on cytoplasmic membrane localization, and is considered to be constitutively active. Thus, while it is unlikely that there are activating mutations in the kinase domain, it is possible that membrane-targeting PDK1 mutations may result in pathway activation (26).

PDK1 is a gene that has been identified as a direct target of miR-375 (27) and is a key component in Akt signaling, a well-documented pathway that regulates cancer cell survival and proliferation.

The overexpression of miR-375 in PC cells may reduce cell proliferation and induce cell apoptosis, suggesting a fundamental role for miR-375 in the development of PC. Accumulating studies have revealed the significance of miRNAs in regulating the growth and apoptosis of cancer cells. miR-21 and -155 have been suggested to function as proto-oncogenes and have been shown to be overexpressed in several cancers (28). miRNA-146a, which is downregulated in various cancer types, has been shown to regulate cell proliferation and apoptosis (29).

A reduction in the expression of miR-375 has been reported in PC (30), hepatocellular carcinoma (HCC) (18) and head and neck squamous cell carcinoma (17). With the exception of its role in cancer, miR-375 is also a significant regulator in mammalian pancreatic islet-cell development and in the regulation of insulin secretion (31), thus indicating its diverse role in normal physiology. The target genes of miR-375 may function cooperatively through different cellular mechanisms. The identification of PDK1 as a target of miR-375 provides new insights into the molecular networks of miR-375. Further studies are required to investigate the targets of miR-375 that may favor the process of tumorigenesis. An introduction of a single miRNA may be beneficial in modulating the complex downstream signals and halting the process of tumorigenesis.

To date, yes-associated protein (YAP) (32), JAK2 (33) and 14-3-3 (27) are other genes that have been identified as direct targets of miR-375. PDK1 has also been identified as a target of miR-375 in esophageal (34) and gastric cancer (35).

The present study identified that the ectopic expression of miR-375 may suppress PDK1 mRNA and protein levels, indicating that miR-375 may also interfere with PI3K-Akt-mTOR signaling. Therefore, miR-375 is a potential therapeutic target against the PI3K-Akt-mTOR signaling axis for preventing PC development and progression.

\section{References}

1. Li D, Xie K, Wolff R and Abbruzzese JL: Pancreatic cancer. Lancet 363: 1049-1057, 2004.

2. Guo $X$ and Cui Z: Current diagnosis and treatment of pancreatic cancer in China. Pancreas 31: 13-22, 2005.

3. Siegel R, Naishadham D and Jemal A: Cancer statistics, 2012. CA Cancer J Clin 62: 10-29, 2012.

4. Hingorani SR, Wang L, Multani AS, et al: Trp53R172H and KrasG12D cooperate to promote chromosomal instability and widely metastatic pancreatic ductal adenocarcinoma in mice. Cancer Cell 7: 469-483, 2005.

5. Bardeesy N, Aguirre AJ, Chu GC, et al: Both p16(Ink4a) and the p19(Arf)-p53 pathway constrain progression of pancreatic adenocarcinoma in the mouse. Proc Natl Acad Sci USA 103: 5947-5952, 2006.

6. Feldmann G, Beaty R, Hruban RH and Maitra A: Molecular genetics of pancreatic intraepithelial neoplasia. J Hepatobiliary Pancreat Surg 14: 224-232, 2007.

7. Westmoreland JJ, Wang Q, Bouzaffour M, et al: Pdk1 activity controls proliferation, survival, and growth of developing pancreatic cells. Dev Biol 334: 285-298, 2009.

8. Bushati N and Cohen SM: microRNA functions. Annu Rev Cell Dev Biol 23: 175-205, 2007.

9. Carthew RW and Sontheimer EJ: Origins and mechanisms of miRNAs and siRNAs. Cell 136: 642-655, 2009.

10. Ambros V: The functions of animal microRNAs. Nature 431: 350-355, 2004.

11. Bartel DP: MicroRNAs: genomics, biogenesis, mechanism, and function. Cell 116: 281-297, 2004.

12. He L and Hannon GJ: MicroRNAs: small RNAs with a big role in gene regulation. Nat Rev Genet 5: 522-531, 2004.

13. Zaman MS, Chen Y, Deng G, et al: The functional significance of microRNA-145 in prostate cancer. Br J Cancer 103: 256-264, 2010.

14. Yu S, Lu Z, Liu C, et al: miRNA-96 suppresses KRAS and functions as a tumor suppressor gene in pancreatic cancer. Cancer Res 70: 6015-6025, 2010.

15. Schickel R, Park SM, Murmann AE and Peter ME: miR-200c regulates induction of apoptosis through CD95 by targeting FAP-1. Mol Cell 38: 908-915, 2010.

16. Szafranska AE, Davison TS, John J, et al: MicroRNA expression alterations are linked to tumorigenesis and non-neoplastic processes in pancreatic ductal adenocarcinoma. Oncogene 26: 4442-4452, 2007.

17. Avissar M, Christensen BC, Kelsey KT and Marsit CJ: MicroRNA expression ratio is predictive of head and neck squamous cell carcinoma. Clin Cancer Res 15: 2850-2855, 2009. 
18. Ladeiro Y, Couchy G, Balabaud C, et al: MicroRNA profiling in hepatocellular tumors is associated with clinical features and oncogene/tumor suppressor gene mutations. Hepatology 47: 1955-1963, 2008.

19. Zhou J, Song S, Cen J, Zhu D, Li D and Zhang Z: MicroRNA-375 is downregulated in pancreatic cancer and inhibits cell proliferation in vitro. Oncol Res 20: 197-203, 2012.

20. Hamilton SR and Aaltonen LA (eds): Pathology and genetics of tumours of the digestive system. In: World Health Organization Classification of Tumours. IARC Press, Lyon, 2000.

21. Wang XQ, Luk JM, Leung PP, et al: Alternative mRNA splicing of liver intestine-cadherin in hepatocellular carcinoma. Clin Cancer Res 11: 483-489, 2005.

22. Wong KF, Luk JM, Cheng RH, et al: Characterization of two novel LPS-binding sites in leukocyte integrin betaA domain. FASEB J 21: 3231-3239, 2007.

23. Liu LX, Lee NP, Chan VW, et al: Targeting cadherin-17 inactivates Wnt signaling and inhibits tumor growth in liver carcinoma. Hepatology 50: 1453-1463, 2009.

24. Kozma SC and Thomas G: Regulation of cell size in growth, development and human disease: PI3K, PKB and S6K. Bioessays 24: 65-71, 2002.

25. Mora A, Komander D, van Aalten DM and Alessi DR: PDK1, the master regulator of AGC kinase signal transduction. Semin Cell Dev Biol 15: 161-170, 2004

26. Storz P and Toker A: 3'-phosphoinositide-dependent kinase-1 (PDK-1) in PI 3-kinase signaling. Front Biosci 7: d886-d902, 2002.

27. El Ouaamari A, Baroukh N, Martens GA, et al: miR-375 targets 3'-phosphoinositide-dependent protein kinase-1 and regulates glucose-induced biological responses in pancreatic beta-cells. Diabetes 57: 2708-2717, 2008.
28. Bloomston M, Frankel WL, Petrocca F, et al: MicroRNA expression patterns to differentiate pancreatic adenocarcinoma from normal pancreas and chronic pancreatitis. JAMA 297: 1901-1908, 2007.

29. Hou Z, Xie L, Yu L, Qian X and Liu B: MicroRNA-146a is down-regulated in gastric cancer and regulates cell proliferation and apoptosis. Med Oncol 29: 886-892, 2012.

30. Lee EJ, Gusev Y, Jiang J, et al: Expression profiling identifies microRNA signature in pancreatic cancer. Int J Cancer 120: 1046-1054, 2007.

31. Poy MN, Eliasson L, Krutzfeldt J, et al: A pancreatic islet-specific microRNA regulates insulin secretion. Nature 432: 226-230, 2004.

32. Liu AM, Poon RT and Luk JM: MicroRNA-375 targets Hippo-signaling effector YAP in liver cancer and inhibits tumor properties. Biochem Biophys Res Commun 394: 623-627, 2010.

33. Ding L, Xu Y, Zhang W, et al: MiR-375 frequently downregulated in gastric cancer inhibits cell proliferation by targeting JAK2. Cell Res 20: 784-793, 2010

34. Li X, Lin R and Li J: Epigenetic silencing of microRNA-375 regulates PDK1 expression in esophageal cancer. Dig Dis Sci 56: 2849-2856, 2011

35. Tsukamoto Y, Nakada C, Noguchi T, et al: MicroRNA-375 is downregulated in gastric carcinomas and regulates cell survival by targeting PDK 1 and 14-3-3zeta. Cancer Res 70: 2339-2349, 2010 . 